\title{
Vingt ans d'histoire du patrimoine architectural et du paysage ferroviaire
}

Twenty Years of the History of Architectural Heritage and Rai lway Landscape

Jean Fosseyeux

\section{OpenEdition}

\section{Journals}

Édition électronique

URL : https://journals.openedition.org/rhcf/1020

DOI : 10.4000/rhcf.1020

\section{Éditeur}

Rails \& histoire

Édition imprimée

Date de publication : 1 juin 2008

Pagination : 245-253

ISSN : 0996-9403

Référence électronique

Jean Fosseyeux, "Vingt ans d'histoire du patrimoine architectural et du paysage ferroviaire ", Revue d'histoire des chemins de fer [En ligne], 39 | 2008, mis en ligne le 01 juin 2011, consulté le 22 avril 2022 URL : http://journals.openedition.org/rhcf/1020 ; DOI : https://doi.org/10.4000/rhcf.1020 
Jean FOSSEYEUX

\section{Vingt ans $d$ 'histoire du patrimoine architectural et du paysage ferroviaire}

1

orsque, il y a maintenant vingt ans, l'Association pour l'histoire des chemins de fer en France s'est constituée, l'existence et l'importance d'un patrimoine bâti ferroviaire français, fortement marqué par une architecture spécifique et donnant naissance à un paysage ferroviaire créé par ce patrimoine et cette architecture ou à partir d'eux, n'étaient perçues que par quelques-uns et depuis assez peu de temps.

Dans le premier numéro de la Revue d'bistoire des chemins de fer, paru en octobre 1989, on trouvait des articles et communications consacrés à l'histoire politique, juridique, administrative, technique, économique, commerciale des chemins de fer, mais le thème du patrimoine n'apparaissait qu'assez furtivement dans une communication de Paul Gerbod sur l'histoire culturelle des chemins de fer en France.

En revanche, dans ce même numéro, figurait, assez discrètement il est vrai, un compte rendu de lecture rédigé par Marie-Noëlle Polino, compte rendu qui concernait l'exposition consacrée aux grandes gares parisiennes du XIX ${ }^{\mathrm{e}}$ siècle, exposition organisée en 1987, année de la constitution de notre association, sous la direction de Karen Bowie qui avait également dirigé la rédaction du catalogue de l'exposition. Dans sa note de lecture, Marie-Noëlle 
Polino rappelait l'exposition, intitulée « le Temps des Gares », présentée dix ans auparavant (en 1978) par le Centre de création industrielle du Centre Pompidou ${ }^{1}$. D'après elle, cette exposition faisait de la gare une utopie et un « ailleurs dans la ville » et elle pensait que l'exposition organisée par Karen Bowie et le catalogue l'accompagnant apportaient une tout autre présentation de la gare, en mettant en lumière les relations des gares :

- avec leurs constructeurs,

- avec les techniques développées aux époques de leur conception,

- avec les quartiers où elles s'implantaient,

- avec les activités et les milieux sociaux de ceux qui empruntaient les trains et qui trouvaient dans ces gares leurs points de départ, d'arrivée ou de changement de direction.

Cette note de lecture rattachant une exposition et son catalogue à une autre exposition et à un autre catalogue lançait ainsi, pour l'AHICF, un thème de recherche qui depuis n’a pas été abandonné.

Avant d'aborder la présentation des principales étapes parcourues par les chercheurs auxquels notre association a permis de s'exprimer, on doit, pour mieux situer l'action de l'AHICF dans le contexte historique de l'époque de sa création, faire une évocation rétrospective de ce qui, avant 1987, s'était passé de marquant dans le domaine qui aujourd'hui retient notre attention.

La première protection au titre des monuments historiques d'un bâtiment ferroviaire remonte à 1926. Elle a concerné l'entrée du tunnel ferroviaire de Fréjus à Fourneaux en Savoie, entrée de tunnel dont on sait qu'elle a été, en 1977, déplacée sur la commune de Modane.

La deuxième protection, toujours une simple inscription, a concerné, en 1938, la gare du Bourbonnais à Lyon, mais on sait que cette gare a été rayée de l'Inventaire en 1953, puis détruite.

En 1965, ce sont trois ouvrages d'art qui ont été inscrits : les viaducs de Garabit, de Rouzat et de Neuvial.

En 1972, un classement a été décidé au profit du buffet de la gare de Lyon et en 1973 est intervenue une décision majeure pour l'histoire des chemins de fer : l'inscription de la gare d'Orsay. Nous pouvons nous arrêter quelques instants sur cette date et sur cette décision qui, à l'époque, détermina de nombreux commentaires. En 1972, la gare d'Orsay n'était plus une gare, du moins plus un «bâtiment voyageurs ». En effet, dès 1938, la jonction souterraine à deux voies reliant les gares d'Orsay et d'Austerlitz s'était révélée

1- Jean Dethier (dir.), Le Temps des gares, catalogue de l'exposition, Paris, éd. du Centre national d'art et de culture G. Pompidou, CCI, 1978, 159 pages. 
saturée aux heures de pointe. Pour le réseau grandes lignes, un autre problème était apparu : les quais d'Orsay ne pouvaient recevoir des trains de plus de douze voitures alors que les nouvelles locomotives pouvaient en tracter seize. La gare de la Belle Époque était de ce fait condamnée et la gare d'Austerlitz redevint le 2 novembre 1939 la tête de ligne du grand réseau. Quant au trafic de banlieue qui restait à Orsay, il fut descendu au sous-sol en 1958. Devenue inapte au service, la gare d'Orsay faillit alors mourir et ses bâtiments disparaître. En 1962 on envisagea réellement d'y envoyer les bulldozers. Un concours d'architecture fut alors lancé en vue d'édifier à sa place un hôtel. Le projet présenté alors par Le Corbusier fut écarté puis le temps passa. Les goûts et les sensibilités évoluant, les débats se développèrent pour savoir s'il fallait ou non démolir cette gare. En 1972, le hall de la gare servait de parking pour les services de la Caisse des dépôts et consignations voisine et abritait le chapiteau de la Compagnie Madeleine Renaud - Jean-Louis Barrault. Un peu plus tard, les commissaires-priseurs rejoignirent l'endroit pour y organiser leurs ventes. Quant à l'hôtel de la gare, appelé Palais d'Orsay, où le général de Gaulle avait prononcé sa célèbre conférence de presse, il fut cette année-là désaffecté. L'année précédente, c'est à dire en 1971, Jacques Duhamel, alors ministre des Affaires culturelles, s'était opposé à l'adoption définitive du projet de grand hôtel destiné à être construit à la place de la gare d'Orsay. En effet ce projet n'avait pas été abandonné. Lentement mais sûrement les autorisations nécessaires avaient été demandées, des financements avaient été rassemblés et le projet avait même reçu un accord préalable du ministre de l'Équipement. Finalement, celui-ci accepta de revenir en arrière et de suivre l'avis tout à fait contraire du ministre de la Culture, avis émis en application de la loi sur les abords des monuments historiques. Le permis de construire fut alors refusé et le ministère de l'Équipement, après plusieurs mois de réflexion, s'orienta non plus vers l'étude d'un nouveau projet de construction neuve mais vers la conservation de la gare d'Orsay et son utilisation par des services publics ou d'intérêt général.

Le premier projet de réutilisation pris en considération fut celui visant à rassembler dans la gare les services du Commissariat au tourisme et différentes « maisons des provinces françaises » installées à Paris. Le ministère de la Culture fit alors valoir qu'il était peu favorable à tout projet d'aménagement conduisant à tronçonner le volume intérieur du bâtiment et que la direction des musées de France pourrait s'intéresser à la gare d'Orsay en vue, par exemple, d'y installer un musée des impressionnistes. C'est dans ce contexte et pour que le ministère de la Culture puisse contrôler l'utilisation de l'édifice que la protection, au titre des monuments historiques, de la gare d'Orsay fut envisagée. 
Les opinions concernant la qualité architecturale de la gare d'Orsay étaient alors assez contrastées mais il était difficile de dénier tout intérêt à cette gare, contemporaine du pont Alexandre III et du Grand Palais, qui s'intégrait très bien dans le site urbain et qui répondait parfaitement aux volumes du Louvre voisin. François Loyer pouvait même à l'époque faire valoir que « la qualité architecturale de la gare lui paraissait supérieure à celle des pavillons terminaux du Louvre » dont l'ornementation était « trop éclectique », Le Fuel s'y étant perdu dans un décor redondant que Visconti aurait su éviter. François Loyer faisait également valoir que le pavillon de Flore avait été construit en 1878 et la gare d'Orsay en 1899. Vingt ans seulement séparaient les constructions des deux bâtiments et il était difficile de croire qu'un édifice nouveau aurait pu mieux s'intégrer dans un contexte aussi exigeant. De son côté, Robert Vassas, tout en reconnaissant les remarquables qualités extérieures et intérieures de la gare, portait un jugement beaucoup plus réservé sur le Palais d'Orsay et regrettait que l'ensemble écrasât trop le délicat Hôtel de Salm. Nonobstant ces réserves, il pensait que le tout devait être préservé.

C'est ainsi que le 12 février 1973 la délégation permanente de la Commission supérieure des monuments historiques proposa l'inscription à l'Inventaire supplémentaire des monuments historiques de la gare en sa totalité et du Palais d'Orsay pour ce qui concernait les façades, les toitures ainsi que la salle des fêtes et la salle à manger avec leurs décors. Quatorze jours plus tard, le 26 février, la commission supérieure entérinait cette proposition. L'événement fut alors abondamment commenté car, en 1973, il était assez rare de protéger un monument du XIX ${ }^{\mathrm{e}}$ siècle. Cette protection était la reconnaissance de l'intérêt que présentaient les grand équipements publics du siècle précédent et l'idée se répandit alors de tenter de faire naitre là ce qu'on avait laissé mourir aux Halles où, pendant quelques temps, s'étaient développés une culture hors commerce et un ludique gratuit.

Le président du conseil d'administration de la SNCF fut formellement informé dès le 8 mars de la protection prononcée. Dans son courrier le ministre lui signalait l'intérêt qu'il y avait à ce que l'État puisse se rendre acquéreur de divers objets mobiliers dignes d'intérêt qui se trouvaient alors dans le Palais d'Orsay. Rapidement, il fut convenu entre le ministère des Affaires culturelles, la SNCF, propriétaire, et le ministère de l'Équipement qui exerçait sa tutelle que l'ancien hôtel du Palais d'Orsay serait affecté aux musées nationaux qui y installerait «les Impressionnistes » puis toute la peinture du XIX ${ }^{\mathrm{e}}$ siècle. Des négociations s'ouvrirent très rapidement pour permettre à l'État d'acheter à la SNCF (qui continuerait à exploiter la gare et ses voies) les locaux abandonnés au début de l'année par l'Hôtel d'Orsay. Le mois suivant, c'est Maurice Druon, 
le nouveau ministre des Affaires culturelles, qui avait remplacé Jacques Duhamel très malade, qui prit en charge le lourd dossier de la gare d'Orsay dans son ensemble. Il ne fallait pas que l'aménagement du futur musée qui serait installé sur l'ensemble de la gare et de l'hôtel soit retardé par les négociations sur le transfert de propriété. En 1977, le président de la République lui-même décidait d'y établir le musée du XIX siècle. En 1978, par un arrêté du 15 mars, la gare et le Palais d'Orsay étaient classés en totalité.

On entra alors dans une assez longue période de querelles étranges autour d'un projet qui avait pourtant semblé assez largement accepté, à savoir : montrer au public l'ensemble des créations d'un siècle riche entre tous et cela dans un bâtiment qui restait l'un des rares et précieux exemples de l'architecture 1900. Ce projet de musée interdisciplinaire, à la fois musée d'art et de civilisation, ne semblait pas rencontrer un acquiescement universel. Certains continuaient à dire qu'ils auraient préféré qu'on ait fait disparaitre cette ancienne gare inutile, incongrue, trop imposante et trop ornée, que l'on ait construit à la place un bel objet architectural, clair et affirmé, et qu'on ait laissé là où elles étaient, éparses et enfouies, les collections que le stupide XIX ${ }^{e}$ siècle avait laissées.

Je ne raconterai pas, après tant d'autres, la longue marche architecturale et muséographique à l'issue de laquelle la gare d'Orsay s'est retrouvée transformée en musée et pour la suite nous irons à très grande vitesse pour rejoindre sans trop tarder la date de 1988, à savoir celle du démarrage de l'AHICF.

Pendant la période allant de 1973 à 1988, ont été protégés, au total, 57 bâtiments ferroviaires. En 1975, se manifesta la première vague de protections, de bâtiments ferroviaires, protections décidées par Michel Guy à l'initiative de Bruno Foucart : trois viaducs et neuf gares auxquels vinrent s'ajouter, en 1976, un viaduc, en 1977, l'ancienne gare de Pierrefonds, en 1981, celle de Cauterêts, en 1982, celle de Valence. La deuxième vague est connue, c'est celle décidée par Jack Lang à l'initiative de Christian du Pavillon. Par trois arrêtés de 1984 furent protégés 19 gares, 12 viaducs, 4 rotondes, 3 têtes de tunnel, 2 funiculaires, 1 château d'eau, 1 maison de garde-barrière.

Revenons donc à 1988, et examinons, à partir de l'exposition sur les gares parisiennes et du catalogue qui l'accompagnait, le développement des recherches historiques conduites à l'initiative de notre association ou avec son soutien. Dès 1990, Karen Bowie rejoignit le comité scientifique de l'AHICF et le premier groupe de travail créé par l'association fut dédié à l'architecture et aux chemins de fer. Il commença aussitôt à se réunir. Au printemps 1991, la quatrième manifestation scientifique de l'AHICF était reçue par le Musée 
d'Orsay : elle s'intitulait «Les chemins de fer et la ville», marquant dès l'abord la dimension urbanistique et sociale que revêtait l'architecture des gares dont le citadin et le voyageur ne perçoivent qu'une partie ${ }^{2}$. Elle démontrait aussi la nécessité de larges comparaisons, dans l'étude de l'architecture ferroviaire, avec des communications qui ont fait date sur les gares d'Europe centrale et d'Italie, et en France même entre plusieurs régions. Notons que cette journée fut ouverte par M. Raoul Balso, historien et ingénieur, qui avait été le dernier chef de gare de la gare d'Orsay et qui est parmi nous aujourd'hui. Le succès de cette journée d'études explique la part importante prise par le thème de l'architecture et du patrimoine dans le colloque organisé en 1993, à Paris, et intitulé «Arts et chemins de fer». La troisième partie de ce colloque avait pour titre "Construire pour le chemine de fer » et comprenait des communications sur :

- les constructions pionnières sur la ligne d'Avignon à Marseille,

- l'architecture ferroviaire et les ouvrages d'art en Île-de-France,

- les gares de l'architecte Louis Brachet sur la ligne de Sceaux,

- la gare du Nord à Paris,

- les gares projetées par Angiolo Mazzoni dans les années 1930-1940.

Le groupe de recherche "architecture et chemins de fer » (aujourd'hui " chemins de fer, architecture et villes »), placé sous la présidence de Karen Bowie, a fourni à l'automne 2000 un ensemble de communications qui ont permis la publication d'un numéro spécial de la Revue d'histoire des chemins de fer consacré à ce que l'on peut appeler l'urbanisme ferroviaire et à l'articulation entre la ville et le réseau ferré. Il comprend des communications portant sur les emplacements de plusieurs gares par rapport aux villes où elles sont implantées : Rennes, Turin, Marseille, Prague, les grandes villes du Rhin supérieur, Strasbourg, mais aussi sur des aspects plus monumentaux et, en particulier, l'escalier de la gare Saint-Charles à Marseille.

En 1998, le thème du patrimoine ferroviaire s'est épanoui à l'occasion du colloque «Le patrimoine ferroviaire : enjeux, bilans et perspectives » organisé au Musée français du chemin de fer de Mulhouse, colloque au cours duquel il fut largement débattu :

- du patrimoine roulant de la SNCF,

- du patrimoine historique de la RATP,

2- RHCF 5-6 [Les références des publications de l'AHICF sont développées dans la bibliographie, p. 325].

3- RHCF 23. 
- de la mise en valeur du patrimoine de la Compagnie internationale des wagons-lits,

- des archives des constructions ferroviaires,

- du patrimoine invisible,

mais aussi :

- de la typologie des gares rurales de moyenne importance du nord-est de la France,

- de la gare de Marseille et de la gare Saint-Lazare à Paris,

- des interventions sur les gares anciennes,

- de la réhabilitation des sites ferroviaires,

- du rôle patrimonial des anciennes voies ferrées.

À la fin de ce colloque, un débat public s'est instauré autour de trois questions concernant la politique du patrimoine ferroviaire en France :

- quels principes?

- quels auteurs?

- quels moyens?

Du débat auquel participèrent des représentants de la ville de Mulhouse, de la SNCF, de RFF (Réseau ferré de France) et de la direction régionale des affaires culturelles sont ressorties quelques idées générales sur:

- les objectifs à partager,

- les responsabilités à définir,

- les moyens à dégager.

Les idées qui se sont alors exprimées mériteraient le développement de nouveaux débats ${ }^{4}$.

La rencontre suivante concernant l'architecture et le patrimoine ferroviaire a été la journée scientifique organisée à Paris le 9 novembre 2006 par le groupe de recherche architecture, chemin de fer et ville, avec pour objet : «Gares et villes, usages et représentations » ${ }^{5}$. Cette journée d'études a permis d'entendre les communications de :

- Stéphanie Sauget sur les usages détournés des gares parisiennes,

- Joanne Vajda sur les Pereire et Nagelmackers, promoteurs du transport ferroviaire et du réseau hôtelier parisien,

- Asta von Buch sur la peinture murale de la gare de Lyon à Paris,

- François Poupardin sur les bâtiments-voyageurs du réseau sud-est de la France,

4- RHCF 20-21.

5- RHCF 38. 
- Frédéric Pillet sur la gare de marchandises des Batignolles,

- Christophe Le Bollan sur l'implantation de la gare de Brest,

- Georges Ribeill sur la délocalisation des installations ferroviaires de Tonnerre à Laroche,

- Thomas Bourelly sur les embranchements particuliers à Lyon au temps du PLM (Paris-Lyon-Marseille),

- Karen Bowie sur la rue Watt et les ponts du Paris-Orléans à Paris.

Il faut également faire état ici des très nombreuses communications présentées à l'occasion d'autres colloques organisés par l'AHICF et concernant peu ou prou l'architecture ou le patrimoine ferroviaires. Certaines de ces communications, dont celle qui m'avait été confiée aux rencontres d'Arles consacrées aux ateliers et dépôts du matériel ferroviaire ${ }^{6}$, ont conduit à élargir les préoccupations de l'association au paysage ferroviaire, souvent consubstantiel avec le patrimoine ferroviaire. Au cours de ces rencontres d'Arles, le double thème des architectures et du patrimoine est réapparu et plusieurs communications furent consacrées à la typologie des dépôts de gare et aux nouvelles rotondes. Enfin, le 3 février 2005, une journée pionnière organisée avec le ministère de l'Écologie et du Développement durable jeta les bases d'une analyse patrimoniale du paysage ferroviaire ${ }^{7}$.

Sur 379 articles publiés dans les numéros 1 à 34 de la Revue d'histoire des chemins de fer, 93 concernent au moins partiellement l'architecture et le patrimoine et le paysage ferroviaire. Si l'on tente de regrouper ces articles par thèmes particuliers, on peut dégager les ensembles suivants :

- les gares et le chemin de fer dans la ville : 30

- les sites et les paysages ferroviaires : 13

- les ateliers et dépôts : 12

- les trains touristiques et la mise en valeur du patrimoine ferroviaire, les chemins de fer et le tourisme : 12

- le cadre juridique, la connaissance et la protection des bâtiments et des sites : 6

- les chemins de fer secondaires: 6

- le rôle de l'architecte, de l'ingénieur et de l'artiste : 5

- les ouvrages d'art : 4

- les voies ferrées : 3

6- RHCF 28-29.

7- RHCF 32-33. 
Au terme de ce survol, avec arrêts sur image, de trente années d'intervention au profit de la connaissance de l'histoire du patrimoine et du paysage ferroviaire, notre association peut s'estimer satisfaite des résultats obtenus. Les travaux concernant le patrimoine et le paysage ferroviaire dont l'AHICF a suscité ou soutenu le développement ont bien trait à l'histoire :

- histoire de la ville et des campagnes,

- histoire des techniques, des savoirs et des métiers,

- histoire des goûts, des pratiques et des échanges,

- histoire des grands événements qui agitèrent le monde et nos sociétés.

Ils ont permis que soient établis des liens fructueux avec d'autres institutions publiques et privées attachées à d'autres titres aux mêmes histoires. C'est donc avec un sentiment de satisfaction pour l'œuvre accomplie et de reconnaissance pour ceux qui ont agi que nous pouvons célébrer ces vingt ans d'existence en écoutant maintenant ceux et celles qui vont pour nous tracer des voies nouvelles pour des recherches et travaux encore plus élargis. 
